\title{
Defining a Sea Ice Flag for Envisat Altimetry Mission
}

\author{
Ngan Tran, Fanny Girard-Ardhuin, Robert Ezraty, Hui Feng, and Pierre Féménias
}

\begin{abstract}
This letter presents the development of a sea ice flag algorithm for the Envisat altimetry mission to detect sea ice corrupted sea surface height data within quality control processing. The algorithm takes advantage of having both passive and active microwave sensors on the same platform with coregistered measurements. Its performances have been evaluated based on collocations between the along-track Envisat data with reference maps built from combination of daily grids of sea ice concentration from SSM/I sensors and backscatter cross section from SeaWinds scatterometer on QuikSCAT satellite.
\end{abstract}

Index Terms-Altimetry, microwave measurements, remote sensing, sea ice.

\section{INTRODUCTION}

$\mathbf{S}$ ATELLITE altimetry is nowadays a standard tool for observing open ocean circulation and its change with time. It provides precise measurements of sea surface topography down to $1 \mathrm{~cm}$. This high level of accuracy is obtained by careful quality control of the measured data. However, as Envisat altimetry mission coverage is up to $81.5^{\circ} \mathrm{N}$ and $\mathrm{S}$, at high latitudes, both altimeter range and wet tropospheric correction computed from radiometer brightness temperature (TB) measurements are corrupted by the presence of sea ice within the respective instrument's footprint. It is thus necessary that such corrupted data be edited out, but currently, there is no specific flag available within Envisat altimetry products to help perform such a task.

Nowadays, the detection of contaminated data is performed by users with their own developed algorithms with more or less success. Most of them are based on the waveform peakiness parameter. Normal ocean returns are expected to have peakiness values in the range of 1.5-1.8 while higher values are observed for specular returns from sea ice [1]. The algorithm of Faugère et al. [2], for instance, relies on a set of thresholds on three parameters: 1) the number of elementary $18-\mathrm{Hz}$ valid measurements effectively used in the processing chain for calculating the standard $1-\mathrm{Hz}$ averaged value of ocean range; 2 ) the Ku-band waveform peakiness; and 3) the difference between model and radiometer-derived wet tropospheric corrections.

Manuscript received July 3, 2008; revised July 28, 2008 and August 13, 2008. First published December 2, 2008; current version published January 14, 2009. This work was supported by the European Space Agency.

N. Tran is with the Space Oceanography Division, Collecte Localisation Satellites (CLS), 31520 Ramonville St-Agne, France (e-mail: ntran@cls.fr).

F. Girard-Ardhuin and R. Ezraty are with the IFREMER, Technopole Brest-Iroise, 29280 Plouzané, France.

H. Feng is with the Ocean Process Analysis Laboratory, University of New Hampshire, Durham, NH 03834 USA.

P. Féménias is with the European Space Research Institute for Earth Observation (ESRIN), European Space Agency, 00044 Frascati, Italy.

Digital Object Identifier 10.1109/LGRS.2008.2005275
The algorithm of Lillibridge et al. [3] is based on a 2-D histogram of radar dual-frequency backscatter cross sections to detect both rain and sea ice corrupted data. The detection capabilities of these algorithms have only been validated, from our knowledge, with respect to global seasonal variations.

In this letter, we present a classification-based algorithm to define a sea ice flag over the arctic region. Several combinations of parameters from the altimeter and the radiometer have been assessed for the development of different classifiers.

The analysis is laid out as follows. Section II provides an overview of Envisat microwave measurements along with a presentation of the collocations performed between Envisat data and referenced sea ice maps. These maps have been built from a combination of SSM/I and SeaWinds daily grids provided by Ifremer/CERSAT. Because of the 1-D Envisat ground track, complete overview of the polar region is obtained over a 35-day period during which sea ice distribution varies with time due to processes of formation, motion, and melting. The collocation data set is therefore used for the following three purposes: 1) to interpret the geographic distribution of sea ice within each Envisat cycle; 2) to validate; and 3) to compare results from the different developed classifiers. Section III focuses on the unsupervised clustering technique and classifier development. Performance assessment of the developed classifiers in terms of good sea ice detection is presented in Section IV.

\section{Data Sets}

\section{A. Envisat RA-2 and MWR Data}

The Envisat platform, launched in March 2002, carries a radar altimeter (RA-2) and a microwave radiometer (MWR). RA-2 is a dual-frequency radar that operates at Ku-band $(13.575 \mathrm{GHz})$ and at S-band $(3.2 \mathrm{GHz})$. The estimation of geophysical quantities is done on-ground by retracking the measured echo waveforms. The backscatter cross section $\left(\sigma_{0}\right)$ data used here are from the "ocean" retracking algorithm.

The MWR is a dual-channel Dicke-type radiometer operating at 23.8 and $36.5 \mathrm{GHz}$. Its primary mission is to determine tropospheric water vapor path delay correction for RA-2 over ocean. Its two TB measurements are provided everywhere. $\mathrm{TB}, \sigma_{0}$, and other Envisat parameters come from standard Geophysical Data Record products [4] spanning the period of year 2004, i.e., from cycles 023 to 033.

\section{B. Daily Sea Ice Maps}

Our validation approach takes advantage of the availability of two daily products generated and distributed by Ifremer/ CERSAT: 1) polar stereographic grids of sea ice concentration (i.e., fraction of the sea surface covered by ice) from SSM/I 
sensors [5] and 2) grids of backscatter cross section from SeaWinds scatterometer on QuikSCAT mission (so-called QSCAT polar sea ice grids) [6]. These grids have a nominal cell size of $12.5 \mathrm{~km} \times 12.5 \mathrm{~km}$. The combination of the two products enables us not only to monitor sea ice extent but also to categorize arctic polar sea ice in terms of the following: multiyear ice (MYI) and consolidated first-year ice (FYI) during winter months (from January to April and from October to December).

MYI has, by definition, survived at one year cycle of melting. It is an old ice as thick as $3 \mathrm{~m}$, whereas FYI has been formed during the current freezing season, developing from young ice and of thickness typically between $30 \mathrm{~cm}$ and $2 \mathrm{~m}$. Such a discrimination is possible because scatterometer $\sigma_{0}$ measurement coming from sea ice depends on ice type. In fact, surface of MYI is rougher than FYI and thus backscatters more signal. This latter is also enhanced from volume scattering in MYI due to deeper radar wave penetration into the cold medium.

Effective discrimination between ice types by scatterometry is only possible in winter when the surface properties affecting $\sigma_{0}$ are stable. Other parameters, such as snow cover, ice temperature, and air humidity, also influence the measured $\sigma_{0}$. During the melting season, water ponds on top of the sea ice cover change values of $\sigma_{0}$, and its distribution map no longer allows an effective discrimination of sea ice type. Therefore, for the period from May to September, there is a simpler indicator separating ice-free ocean and "global" sea ice (GSI) in the derived maps. Note that over ice-free ocean, $\sigma_{0}$ depends on surface roughness due to winds.

The derivation of the daily polar sea ice maps includes several steps, leading to the definition of a seven-state flag that separates: 1) coastal (areas of three pixels with no reliable values of sea ice concentration along the land mask); 2) ice-free ocean; 3) FYI; 4) MYI; 5) GSI; 6) land; and 7) ambiguous pixel.

Setting the flag as an ambiguous pixel relies on the application of four successive filters on each built daily grid. The first one performs an automatic detection of suspicious ice edge grid points (area delineated by the $0 \%$ and $15 \%$ ice concentration contours). Then, a statistical yearly mask is applied to point out pixels with less than six occurrences of sea ice during the whole year. This mask (not shown) allows the highlight of small areas in open ocean, i.e., far off the sea ice pack or along midlatitude coastlines, which are related for most of them to atmospheric artefacts inducing misidentifications as sea ice. The third screening consists in applying a three-day filter on short-term lived sea ice pixels, i.e., grid points where there are short-term changes in category identification related to ice formation or motion. Application of these three filters on the daily grids produces "cleaner" maps since if we built afterward a new sea ice mask over the year period, we obtain mainly a big sea ice pack with only a few and very small areas away from it. We add then a fourth filter based on geographical boundaries to dismiss all small isolated areas in the daily maps. The combination of these different filters sets the flag to the ambiguous state for $\sim 3.7 \%$ of the grid data points over the oneyear period. This cleaning procedure allows us to consider only the grid points with high confidence in category identification. These derived sea ice maps are then used to provide category at each Envisat position through a collocation procedure.
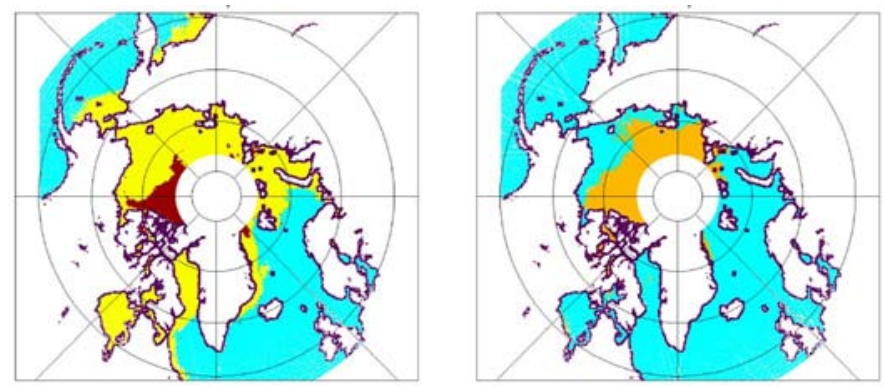

Fig. 1. Collocated MCSQ data maps over Arctic region from cycles 025 (winter situation) and 029 (summer situation). Each map displays sampling from one Envisat 35-day cycle (blue: Ice-free ocean, yellow: FYI, brown: MYI, and orange: "GSI").

\section{Collocated Data}

The collocation procedure consists in a bilinear spatial interpolation, i.e., the sea ice map values for a given day are interpolated to the Envisat positions observed on the same day. The evaluation of Envisat sea ice detection performances will be performed only on the "clean" interpolated data, i.e., displaying the same category (or value) as all four surrounding data grid points used for the interpolation. This leaves out Envisat data located primarily near the margins between two categories on the sea ice map since it is difficult to sort out if an Envisat position should be associated to one category or to the other with high confidence. This editing removes 30.4\%-32.3\% of the collocated data per Envisat cycle. This percentage is higher for winter cycles because the sea ice pack has a more extensive coverage and therefore has a longer boundary line. Fig. 1 shows two examples of cyclic map of the "clean" collocated data. They are referred as merged and collocated SSM-I/ QSCAT maps (MCSQ) in the following. These MCSQ data will serve to label the different clusters within a set determined by an unsupervised clustering method which is applied only on Envisat data. Each set defines a classifier solution. The MCSQ data are used afterward to evaluate the performances of the different developed classifiers.

\section{Classification Algorithm}

Several techniques are available for classifying objects within an $N * P$-dimensional data set. We use hereafter an unsupervised iterative clustering approach that searches for natural clusters in the data set. This partition of the objects (characterized by several features) into clusters (also called classes) consists in grouping objects that have similar features between themselves and are dissimilar to objects in other clusters. Each cluster is characterized by a center in $\Re^{\mathrm{P}}$. The followup task is to label each of the resultant "unnamed" clusters with identified category related to the sea ice type. Representing data by fewer clusters necessarily looses certain fine details but achieves simplification.

The clusters in the data set are usually not completely well separated. Fuzzy clustering is a popular method for dealing with this issue. It computes gradual memberships and allows objects to be associated with more than one cluster at the same time by assigning to each object a degree of membership to each cluster. 
A membership is computed from the distance of the object to a given cluster center. The closer an object is to a center, the higher is its degree of membership to the associated cluster. It also allows the interpretation of the results in a "hard" partition way, i.e., associating each object to only one unique class by using the highest membership value as a first-order evaluation. Therefore, the results from such an approach can easily be interpreted at different levels of information with respect to need of details. Among the fuzzy methods, fuzzy c-means (FCM) iterative clustering algorithm is, by far, the most popular one [7], [8] and is frequently used in pattern recognition [9].

We applied in the following the FCM clustering algorithm to the collocated Envisat data. The algorithm generates a classification of the data into a prespecified number of clusters $c$. Ideally, this number $c$ should correspond to the number of substructures naturally present in the data set.

\section{A. FCM Clustering}

Each classifier is developed based on a data set that has $N$ objects $\left\{\overrightarrow{x_{1}}, \ldots, \overrightarrow{x_{N}}\right\}$ which are individually described by $P$ features. The FCM clustering algorithm partitions this collection of objects into $c$ clusters with subscript $i$ ranging from 1 to $c$ and finds a $P$-dimensional vector $\vec{\nu}_{i}$ corresponding to the center, in each group. The locations of the different centers are adjusted iteratively after a randomized initialization of the vectors $\vec{\nu}_{i}$ and until optimization criteria are met by minimizing the objective function $J$ given by

$$
J=\sum_{i=1}^{c} \sum_{k=1}^{N} u_{i k}^{m} d_{i k}^{2}=\sum_{i=1}^{c} \sum_{k=1}^{N} u_{i k}^{m}\left\|\overrightarrow{x_{k}}-\overrightarrow{\nu_{i}}\right\|^{2} .
$$

In this expression, $u_{i k} \in[0,1]$ represents the membership degree of object $\overrightarrow{x_{k}}$ in the $i$ th cluster represented by $\vec{\nu}_{i}$ and satisfies the following constraint:

$$
\sum_{i=1}^{c} u_{i k}=1 \quad \forall k=1, \ldots, N .
$$

$c$ and $m$ are two parameters that have to be specified before running the algorithm. $m$ is a weighting exponent which controls the fuzziness of the method. This parameter states how much the clusters are allowed to overlap. $d_{i k}^{2}$ in (1) is the squared Euclidian distance between object $\overrightarrow{x_{k}}$ and cluster center $\overrightarrow{\nu_{i}}$. At each iteration, the two following functions are updated in order for $J$ to reach a minimum

$$
\begin{aligned}
\overrightarrow{\nu_{i}} & =\frac{\sum_{k=1}^{N} u_{i k}^{m} \overrightarrow{x_{k}}}{\sum_{k=1}^{N} u_{i k}^{m}} \quad \forall i=1, \ldots, c \\
u_{i k}= & \frac{1}{\sum_{j=1}^{c}\left(\frac{d_{i k}}{d_{j k}}\right)^{2 /(m-1)}} \\
& \forall i=1, \ldots, c \text { and } \forall k=1, \ldots, N .
\end{aligned}
$$

The iteration process stops when predefined convergence criterion is met, i.e., that the coordinates of the cluster centers remain unchanged $(<\varepsilon)$ from one iteration to the next. Values of
TABLE I

Statistical Characteristics, Mean, AND Standard DeViation of SEVEn EnVis at PARAMETERS. THEy ARE CoMPUted From DATA OVER THE ARCTIC POLAR REGION From CYCLES 025 AND 029 (RT: RATION_TB)

\begin{tabular}{lccccccc}
\hline \hline & $\begin{array}{c}\text { avg_TB } \\
(\mathrm{K})\end{array}$ & $\begin{array}{c}\mathrm{Ku \_} \sigma_{0} \\
(\mathrm{~dB})\end{array}$ & $\begin{array}{c}\Delta \mathrm{TB} \\
(\mathrm{K})\end{array}$ & $\begin{array}{c}\mathrm{RT} \\
(-)\end{array}$ & $\begin{array}{c}\mathrm{S}_{-} \sigma_{0} \\
(\mathrm{~dB})\end{array}$ & $\begin{array}{c}\text { Ku_peak } \\
(-)\end{array}$ & $\begin{array}{c}\text { S_peak } \\
(-)\end{array}$ \\
\hline mean & 192.87 & 15.82 & 4.82 & 0.016 & 15.58 & 9.54 & 6.16 \\
std & 36.94 & 8.00 & 10.77 & 0.03 & 6.10 & 13.41 & 6.87 \\
\hline \hline
\end{tabular}

optimized membership matrix $\mathbf{u}$ and cluster center coordinates' matrix $\mathbf{v}$ are then obtained for the developed classifiers.

\section{B. Development of Envisat Classifiers}

We developed 11 classifiers. Ten of them are two-parameter classifiers, and one is a three-parameter classifier in order to test different combinations of the seven selected input parameters, including the following: 1) average of the two TBs avg_TB; 2) Ku-band backscatter $\mathrm{Ku}_{-} \sigma_{0}$; 3) difference between the two TBs $\Delta$ TB (i.e., TB_36.5 - TB_23.8); 4) spectral ratio of the TBs, ratio_TB, defined as (TB_36.5 - TB_23.8)/(TB_36.5 + TB_23.8); 5) S-band backscatter $\mathrm{S}_{-} \sigma_{0}$; 6) Ku-band peakiness Ku_peak; and 7) S-band peakiness S_peak.

Table I summarizes their statistical characteristics. Different parameter ranges of variation require a data standardization to ensure that each parameter is given appropriate weight in the classifier development. We used the standard approach by shifting and scaling the values so that each of the input parameters under consideration has zero mean and unit variance. This is done by using values provided in Table I. Two cycles of data have been used to develop the algorithm: a winter one (cycle 025) and a summer one (cycle 029), covering the two periods from March 9 to April 12 and from July 27 to August 30, respectively. This data merging is done to get a global representation of the different microwave signatures containing seasonal variability.

Fuzzifier $m$ is an important parameter for FCM. Most of the literatures recommend choosing $m$ around two. Here, it is set to two. The number of class $c$ is specified a priori based on the sea ice categories that we are looking for, i.e., two for the present analysis to separate ice-free ocean pixels from sea ice contaminated ones. However, since the clustering approach is unsupervised and looks for natural subsets in the data set, it appears after several attempts that the iterative algorithm converges to stable solutions, i.e., independent of the initialization of the cluster center locations when $c$ is set to four. This is clearly shown in Fig. 2. The distributions of open water and sea ice data groups in two different two-parameter diagrams, for instance, do not display the same extents and localizations when one compares the winter and the summer situations. In order to take into account this seasonal variability in signatures, four cluster centers are needed to correctly describe the distinct substructures present in the data set.

Different possibilities occur afterward for the labeling of these cluster centers. Either each one of them represents a distinct sea ice category or combined use of some of them is 

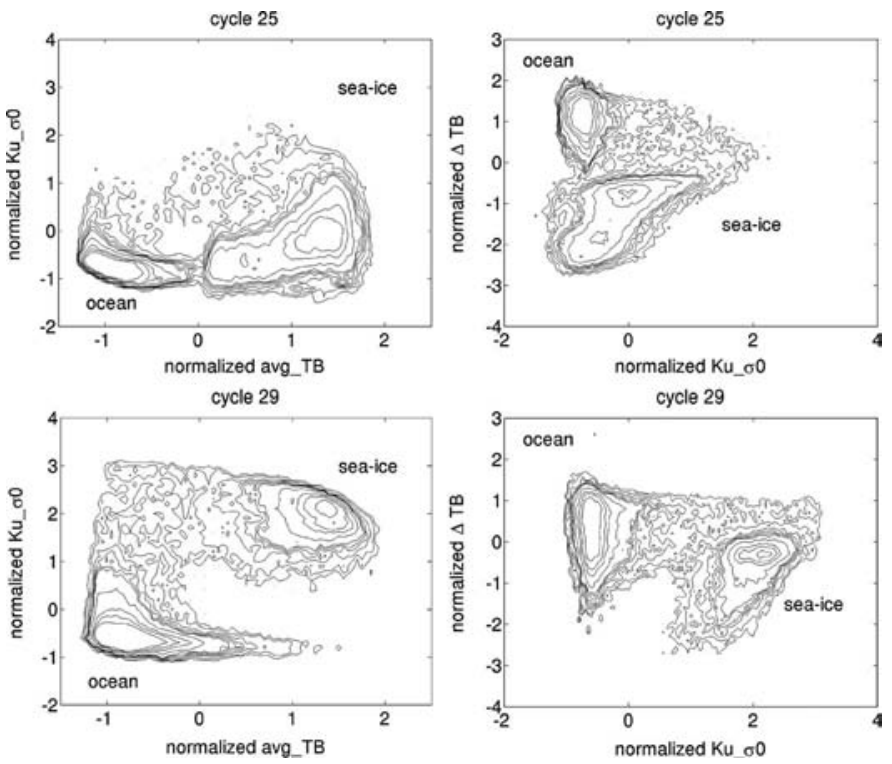

Fig. 2. Distribution of data from cycles 025 and 029 in the normalized 2-D plan of (avg_TB, Ku_ $\sigma 0)$ and $\left(\mathrm{Ku} \_\sigma 0, \Delta \mathrm{TB}\right)$. The contours represent isodensity distribution of the data when projected in the respective plan.
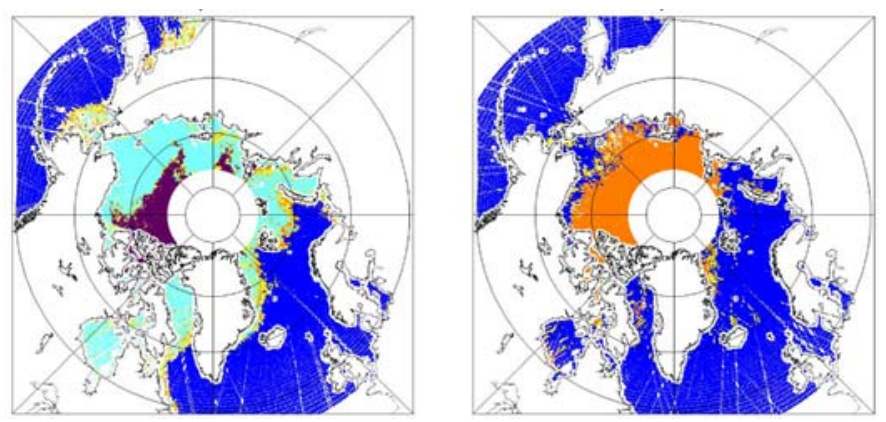

Fig. 3. Maps of Envisat three-parameter classifier results for cycles 025 and 029 data, respectively. The map displays sea ice category distributions (dark blue: Ice-free ocean, light blue: FYI, purple: MYI, orange: Wet ice, and yellow: Ambiguous).

required to globally define a specific cluster. This labeling of the cluster centers is performed based on comparative features between MCSQ maps in Fig. 1 and maps of the three-parameter classifier result distribution shown in Fig. 3 for instance. In this case, each cluster center seems to be associated to a specific sea ice type, and these four classes are the following: 1) ice-free ocean; 2) FYI; 3) MYI; and 4) wet ice. Table II provides the coordinates of cluster centers for this three-parameter classifier in the standardized parameter space. In Fig. 3, one can see that cluster 1 is associated to ocean data, cluster 2 to FYI, cluster 3 to wet sea ice, and cluster 4 to MYI.

Concerning the two-parameter classifiers, distinction of icefree ocean pixels from sea ice ones requires the combination of two clusters to describe each category.

\section{RESUlts}

For each Envisat position, one classifier provides a set of membership values associated to corresponding cluster centers. When the highest membership is at least 0.55 , we associate the label of the corresponding cluster center to the Envisat data since it represents the dominant sea ice category in the sensors'
TABLE II

CoOrdinates of THE Cluster CEnTERs Associated to THE THREe-PARAMETER Classifier IN THE NORMALIZED PARAMETER SPACE AS DENOTED B Y THE SUBSCRIPT $N$ AND CORRESPONDENCE IN TERMS OF SEA ICE ClASS

\begin{tabular}{lccc}
\hline \hline & avg_TB & $\mathrm{Ku}_{\mathrm{n}} \sigma_{0 \mathrm{n}}$ & $\Delta \mathrm{TB}_{\mathrm{n}}$ \\
\hline cluster 1 : ocean class & -0.8063 & -0.5322 & 0.7052 \\
cluster 2 : FYI class & 1.1820 & 0.0120 & -0.8588 \\
cluster 3 : wet ice class & 1.2050 & 2.0123 & -0.4735 \\
cluster 4 : MYI class & 0.5448 & -0.4639 & -1.9484 \\
\hline \hline
\end{tabular}
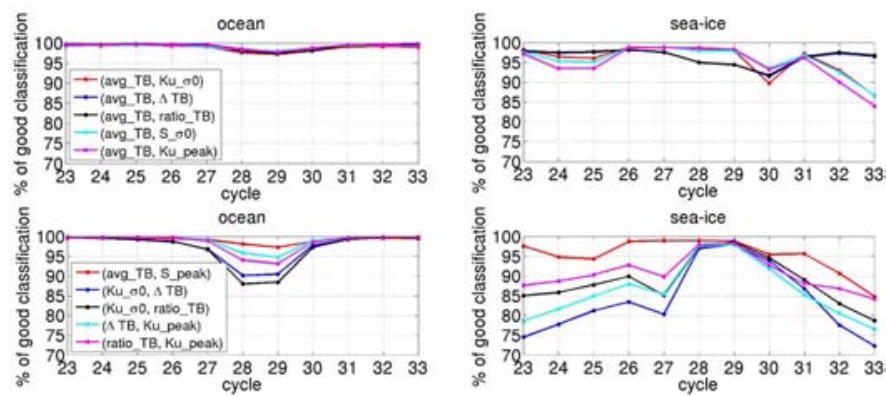

Fig. 4. Performances of Envisat two-parameter classifiers as a function of cycle number and in terms of good classification of (left panels) ice-free ocean pixels and (right panels) sea ice contaminated pixels. The MCSQ flag is used as reference.

fields of view. When the highest membership is lower than 0.55 , the Envisat data at this location are indicated as ambiguous (plotted in yellow in Fig. 3) with some mixture of sea ice categories within the fields of view.

To validate the sea ice detection results for the different classifiers, the MCSQ flag is used as a reference. Recall that the MCSQ information is based on daily grids of statistical characteristics while the Envisat algorithm works on a measurement pixel basis, and therefore, the results from the latter are expected to be slightly noisier than those from the former. Note also that since the purpose of the classifier development is to edit sea ice contaminated data for ocean altimetry applications, we will only discuss the good detection of ice-free ocean data and sea ice data (i.e., by merging FYI, MYI, wet ice types, and ambiguous data into one group) hereafter. The performance assessment of the algorithms in identifying MYI, FYI, and wet ice data is out of the scope in this letter. On the other aspect, these categories of sea ice aforementioned do provide explanations on the fact that $c$ has to be set to four in order to determine stable classification solutions.

Figs. 4 and 5 show the time series of classifiers' performances in terms of good identification when compared with the categorization provided by MCSQ. Fig. 4 shows results for the ten two-parameter algorithms. For data identified by Envisat as ice-free ocean, the percentages of good classification are higher than $85 \%$ for all algorithms. They are stable particularly over the winter period when there appear percentages higher than $95 \%$. All of them display a decrease in performances for cycles 28-30 (late June to beginning of October) during the summer season when the percentages vary between $87 \%$ and $98 \%$ depending on the two-parameter combination being used. 

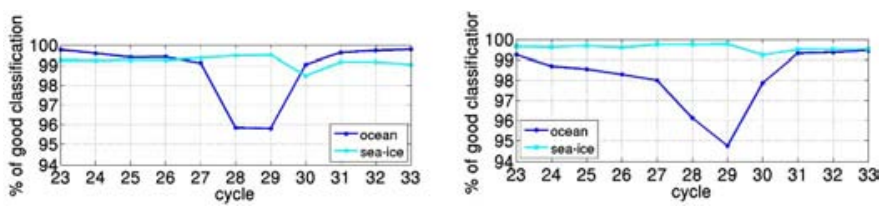

Fig. 5. Performances of (a) the Envisat three-parameter classifier and (b) the algorithm of Faugère et al. [2] as a function of cycle number. The MCSQ flag is used as reference.

Fig. 4 also shows the percentages of good identification of sea ice data when compared with the MCSQ flag values corresponding to FYI, MYI, or GSI. The percentages are above $70 \%$ and display much more variation with respect to the pair of parameters tested and the cycle analyzed. The classifier based on avg_TB and $\triangle \mathrm{TB}$ parameters (from radiometer only) provides the best overall performance for the ice-free ocean and sea ice detections if one considers only the winter cycles.

In Fig. 5, Envisat three-parameter classifier shows good and stable performances during the winter season with more than $99 \%$ of good identification of either ice-free ocean or sea ice data (all cycles, except for cycle 30). Lower percentages in the identification of ice-free ocean data are observed from mid-June to end of August (cycles 028 and 029) around 95.8\%. In fact, a detailed analysis shows that $\sim 2 \%$ of the misclassified ice-free ocean data were identified by Envisat classifier as belonging to the mixed class of open water and sea ice that we have included in the sea ice group. Performances of the ice flag algorithm proposed by Faugère et al. [2] are also provided for comparison. Percentages of good sea ice detection are higher than $99 \%$ for the whole 2004 year. Concerning the ice-free ocean detection, it showed that the percentage of good identification is larger in winter than in summer. Indeed, it is higher than $98 \%$ in winter, whereas during summer months from May to September (cycles 27-30), the value is lower, namely, between $94.5 \%$ and $98 \%$. The sea ice algorithm developed by Faugère et al. [2] displays, overall, very good performances, similar to those from our three-parameter classifier.

\section{Summary AND CONCLUSION}

In this letter, we presented several classifiers to retrieve sea ice category over Arctic polar region by combinations of active and passive coregistered data from Envisat altimetry mission. Results show good performances of the present approach for recognition of sea ice corrupted data versus ice-free ocean data when compared with combined SeaWinds and SSM/I records. Overall, performance of the three-parameter classifiers is slightly better than those of the two-parameter classifiers under evaluation. This latter can help oceanographic users to edit sea ice contaminated sea surface height data. A flag derived from this classifier will be made available in the coming soon reprocessed products of Envisat altimetry mission.

Another quality of this classifier is that its set of input parameters makes such development applicable to all past and future altimetry missions since such configuration of the instruments, i.e., with monofrequency altimeter and dual-frequency radiometer, represents the baseline requirement. Indeed, these missions do not always embark dual-frequency altimeters as shown by ERS missions and next coming AltiKa altimeter on Saral mission. Concerning the other tested parameters, they are not always available on other missions, or anomalies on some of them have been reported. For instance, Envisat RA-2 S-band data sometimes display some on-board anomalies at the latest happening in January 2008 with a sudden drop of the transmission power. The peakiness parameter used in the algorithm of Faugère et al. [2] is currently only available in Envisat products while RA-2 Ku-band backscatter and MWR TBs used in our three-parameter classifier represent the basic and primary retrieved geophysical parameters from raw data for all altimetry missions.

Further works would be related to the interpretation of the ambiguous data points related to a mixture of categories and of their proportion with respect to the threshold on membership that we have fixed here at 0.55 to identify "dominant" category for a measurement location. Mixture occurrences look realistic when one considers the large footprint sizes of the Envisat sensors.

\section{ACKNOWLEDGMENT}

The authors would like to thank T. S. Moore who kindly provided his FCM routines which form the basis of the classification development.

\section{REFERENCES}

[1] S. W. Laxon, "Sea ice altimeter processing scheme at the EODC," Int. J. Remote Sens., vol. 15, no. 4, pp. 915-924, Mar. 1994.

[2] Y. Faugère, F. Mertz, and J. Dorandeu, "Envisat validation and cross calibration activities during the verification phase," in "Synthesis Report," 2003. Technical Note CLS.DOS/NT/03.733, ESTEC contract No16243/02/NL/FF, WP6. [Online]. Available: http://earth.esa.int/pcs/ envisat/ra2/articles/Envisat_Verif_Phase_CLS.pdf

[3] J. Lillibridge, R. Scharroo, and G. Quartly, "Rain and ice flagging of Envisat altimeter and MWR data," in Proc. Envisat ERS Symp., Salzburg, Austria, 2004. [Online]. Available: http://eprints.soton.ac.uk/17318/

[4] J. Benveniste, S. Baker, O. Bombaci, C. Zeli, P. Venditti, O.-Z. Zanife, B. Soussi, J.-P. Dumont, J. Stum, and M. P. Milagro-Perezin Envisat RA-2/MWR Product Handbook. Frascati, Italy: Eur. Space Agency, 2002. PO-TN-ESR-RA-0050. [Online]. Available: http://envisat.esa.int/ dataproducts/ra2mwr/CNTR.htm

[5] R. Ezraty, F. Girard-Ardhuin, J. F. Piollé, L. Kaleschke, and G. Heygsterin "Arctic and Antarctic sea ice concentration and Arctic sea ice drift estimated from Special Sensor Microwave (SSM/I), User's manual," Tech. Rep. C2-MUT-W-07-IF, Feb. 2007. Version 2.1, DOPS/LOS/IFREMER. [Online]. Available: http://www.ifremer.fr/cersat

[6] R. Ezraty and J. F. Piollé, "SeaWinds on QuikSCAT polar sea ice grids User's Manual," Convection Report no5, DOPS/LOS/ IFREMER, 2001. Tech. Rep.

[7] F. Wang, "Fuzzy supervised classification of remote sensing images," IEEE Trans. Geosci. Remote Sens., vol. 28, no. 2, pp. 194-201, Mar. 1990.

[8] T. S. Moore, J. W. Campbell, and H. Feng, "A fuzzy logic classification scheme for selecting and blending satellite ocean color algorithms," IEEE Trans. Geosci. Remote Sens., vol. 39, no. 8, pp. 1764-1776, Aug. 2001.

[9] J. C. Bezdek, Pattern Recognition With Fuzzy Objective Function Algorithm. New York: Plenum, 1981. 